\title{
LETTERS
}

\section{What constitutes a public health response to the opioid overdose epidemic?}

I read with interest Dr. Tyndall's recent commentary in CMAJ, ${ }^{1}$ which calls for the use of a regulated, low-barrier distribution of pharmaceutical-grade opioids to address the immediate challenges extant to today's opioid overdose epidemic. Of concern, however, is the statement that "the public health response to any poisoning epidemic should be to provide safer alternatives for people at risk." This statement follows the preceding paragraph stating that a number of "upstream" interventions are "critical to our response," but that the outcomes of these are longer term and - to paraphrase - aren't meeting the immediate need.

The first thought that occurred to me was whether the words "should include" would be preferable to "should be." Indeed, to distill a complex problem that requires a comprehensive public health response into a single downstream intervention misunderstands the basic function of public health practice. Additionally, the pieces Dr. Tyndall identifies as being "upstream" but too far away on the time horizon constitute key elements of a public health response.
Public health's response to the opioid overdose epidemic must continue to be multifaceted. On an ongoing basis, the response is built on effective surveillance and evaluation to describe the shape and size of the issue and monitor the outcomes of interventions. Layered onto this is partnership with the health care system and various community agencies to address the present mortality and morbidity among those who choose to use opioids, which can include possible deployment of the single novel approach proposed by Dr. Tyndall.

Finally, the work and advocacy of public health with relevant stakeholders (government, housing, social services, law enforcement and others) will be most critical in reversing the epidemic; although these have a long time horizon, they are what will ultimately address the antecedent factors that contribute to opioid use and poor mental health.

To be clear, I agree with Dr. Tyndall to some extent - a public health response to any poisoning epidemic should include providing safer alternatives for people at risk. But it is important not to minimize the other elements of that response simply because they are difficult or lack immediacy. Our progress in reducing deaths from lack of road safety was not owing to improved postaccident trauma care alone. Our successes to date with tobacco were not solely because of the provision of nicotine replacement therapy. Our efforts with respect to alcohol do not revolve around light beer alone.

Hence, in calling something a public health response, particularly an issue as prominent as the opioid overdose epidemic, I would propose that due recognition be given to the complex nature of both the problem and solutions, with an acknowledgement that advocacy for response and downstream interventions should not be conducted at the expense of those broader population interventions that will ultimately resolve this crisis.

\section{Lawrence C. Loh MD MPH}

Adjunct professor, Dalla Lana School of Public Health, University of Toronto, Toronto, Ont.

Cite as: CMAJ 2018 May 28;190:E661. doi: $10.1503 /$ cmaj.69272

\section{Reference}

1. Tyndall M. An emergency response to the opioid overdose crisis in Canada: a regulated opioid distribution program. CMAJ 2018;190:E35-6.

Competing interests: None declared. 